\title{
Insatisfação com os serviços odontológicos entre idosos brasileiros dentados e edentados: análise multinível
}

\author{
Dissatisfaction with dental services among dentate \\ and edentulous elderly Brazilians: a multilevel analysis
}

\author{
João Gabriel Silva Souza ${ }^{1}$ \\ Bárbara Emanoele Costa Oliveira ${ }^{1}$ \\ Carolina Veloso Lima ${ }^{1}$ \\ Aline Araujo Sampaio ${ }^{2}$ \\ Mayara dos Santos Noronha ${ }^{1}$ \\ Renata Francine Oliveira ${ }^{3}$ \\ Efigênia Ferreira e Ferreira ${ }^{2}$ \\ Andrea Maria Eleutério de Barros Lima Martins ${ }^{3}$
}

${ }^{1}$ Faculdade de Odontologia de Piracicaba, Universidade Estadual de Campinas. Av. Limeira 901, Areião. 13414903 Piracicaba SP Brasil. jgabriel.ssouza@

yahoo.com.br

${ }^{2}$ Faculdade de Odontologia, Universidade Federal de Minas Gerais. Belo Horizonte MG Brasil. ${ }^{3}$ Departamento de Odontologia, Universidade Estadual de Montes Claros. Montes Claros MG Brasil.

\begin{abstract}
The scope of this study was to evaluate dissatisfaction with dental services among dentate and edentulous elderly Brazilians and its association with contextual and individual variables. The data from the National Oral Health Survey conducted in 2010 and contextual variables related to cities were used. Descriptive, bivariate and multilevel analysis was conducted among 1,989 elderly individuals. Of these, $11.2 \%$ of dentate and $22.1 \%$ of edentulous elderly people were dissatisfied. Among the dentate individuals, dissatisfaction was associated with contextual variables (location of municipality, human development index, GINI Index, the presence of Dental Specialty Centers and the coverage of oral health in public service) and persons related to health care and oral health conditions. For the edentulous individuals, no associated contextual variables were identified. Only the motive for the use of the service and satisfaction with teeth and mouth were associated with dissatisfaction with dental services. A considerable prevalence of dissatisfaction with the services was identified, being higher among edentulous elderly Brazilians. Among dentate elderly Brazilians, an association with contextual variables was detected.
\end{abstract}

Key words Patient satisfaction, Dental care, Quality of health care, Elderly
Resumo Avaliou-se a insatisfação com os serviços odontológicos entre idosos brasileiros dentados e edentados e sua associação com variáveis contextuais e individuais. Foram utilizados os dados do Levantamento Nacional de Saúde bucal realizado em 2010 e dados contextuais referentes aos municípios. Análises descritivas, bivariadas e multinivel foram realizadas. Incluiu-se $1.989 \mathrm{ido-}$ sos. Destes, $11,2 \%$ dos idosos dentados e $22,1 \%$ dos edentados estavam insatisfeitos. Entre os dentados, a insatisfação foi associada com variáveis contextuais (localização do município, índice de desenvolvimento humano, Índice de GINI, presença de Centro de Especialidades Odontológicas e cobertura das equipes de saúde bucal) e individuais pertencentes à atenção à saúde e condições de saúde bucal. Para os idosos edentados, não foi identificado associação com variáveis contextuais. Apenas o motivo do uso do serviço e a satisfação com dentes e boca foram associados. Identificou-se uma prevalência considerável de insatisfação com os serviços, sendo maior entre os idosos edentados. Entre os idosos dentados, foi observado associação com variáveis contextuais.

Palavras-chave Satisfação do paciente, Assistência odontológica, Qualidade da assistência à saúde, Idoso 


\section{Introdução}

A satisfação do usuário é considerada um dos principais objetivos a ser alcançado pelos serviços de saúde, sendo também ferramenta importante na avaliação da qualidade do serviço prestado $^{1,2}$. Essa avaliação fornece informações complementares às análises técnicas, sendo uma visão compartilhada da percepção dos usuários que recebem o cuidado ${ }^{3}$. Dessa forma, este indicador torna-se uma importante ferramenta para a gestão do setor de saúde, permitindo delinear estratégias que proporcionem maior eficiência e efetividade ao sistema, atendendo, apropriadamente, as necessidades da populaçã $0^{4,5}$. Nesse sentido, estudos prévios demonstram que a descrição e identificação dos possíveis fatores associados à satisfação ou insatisfação dos usuários com diferentes serviços de saúde são relevantes para reformulações destes serviços de saúde ${ }^{6-10}$.

Assim, torna-se relevante a avaliação dos serviços odontológicos ${ }^{8-10}$, principalmente, ao se considerar a alta prevalência de doenças e agravos bucais na população brasileira ${ }^{11}$, bem como a problemática em volta da oferta e acesso aos serviços odontológicos ${ }^{11,12}$. Ressalta-se que, no Brasil, a insatisfação com os serviços odontológicos tem sido associada à diferentes fatores como, por exemplo, as condições socioeconômicas, a saúde bucal do usuário e algumas características desses serviços $^{8-10}$. Dessa forma, a caracterização dos insatisfeitos, considerando também as condições contextuais, é importante para otimizar a assistência odontológica prestada.

Quando se considera as condições de saúde bucal da população brasileira ${ }^{11}$, a população idosa, estrato etário que cresce exponencialmente no Brasil ${ }^{13}$, merece atenção devido à precariedade das suas condições de saúde bucal e baixo uso de serviços odontológicos em nível nacional ${ }^{11}$. Portanto, a satisfação desses usuários com os serviços odontológicos pode subsidiar informações para compreender questões relacionadas à busca pelo serviço, adesão ao tratamento, às mudanças no estado de saúde e nos comportamentos relacionados a saúde.

Sabe-se que diferenças no uso dos serviços odontológicos e na percepção de saúde bucal parece ser alterada pela presença de dentes na boca $^{14,15}$. No entanto, poucos estudos levam em consideração a condição dentária dos idosos (dentados e edentados) na avaliação da satisfação dos serviços odontológicos. Dessa forma, propõe-se estimar a prevalência da insatisfação com os serviços odontológicos entre idosos brasileiros dentados e edentados, assim como identificar os possíveis fatores associados a esta avaliação considerando variáveis individuais e contextuais dos municípios.

\section{Metodologia}

\section{Amostragem e calibração}

Utilizou-se a base de dados do inquérito nacional das condições de Saúde Bucal da população brasileira (SB Brasil, 2010), seguindo os critérios propostos pela OMS em 1997. Uma amostra representativa da população brasileira nas faixas etárias índices foi entrevistada e examinada em seus domicílios quanto às condições de saúde bucal, demográficas e socioeconômicas, uso de serviços odontológicos e questões subjetivas de saúde bucal. No presente estudo, utilizou-se a amostra de idosos (65 a 74 anos) ${ }^{11}$.

Foram considerados idosos residentes em 177 municípios, incluindo as 27 capitais brasileiras, das cinco macrorregiões brasileiras (Norte, Nordeste, Centro-Oeste, Sudeste e Sul), selecionados por amostragem probabilística por conglomerados, em múltiplos estágios, com probabilidade proporcional ao tamanho e considerando um efeito de desenho (deff) igual a 2. A seleção amostral foi estruturada em dois estágios para as capitais e Distrito Federal e em três estágios para os municípios do interior das cinco macrorregiões brasileiras. Os 30 municípios em cada região e 30 setores censitários para capitais e Distrito Federal foram sorteados pela técnica de Probabilidade Proporcional ao Tamanho ${ }^{11,16}$.

Os exames e entrevistas foram realizados por cirurgiões-dentistas previamente treinados e calibrados pela técnica do consenso, sendo o valor mínimo aceitável de kappa para cada examinador, grupo etário e agravo estudado igual a 0,61 . As entrevistas foram realizadas com auxílio de computador de mão (Personal Digital Assis$\operatorname{tant})^{16}$.

Para este estudo, foi feito um recorte do banco de dados, e foram incluídos nas análises os idosos que relataram ter utilizado o serviço odontológico há menos de 1 ano para minimizar o viés de memória. A avaliação da satisfação com o serviço odontológico foi feita em dois estratos: dentados e edentados. Para diferenciação dos idosos dentados e edentados, utilizou-se o componente " $\mathrm{P}$ perdido" do CPO-D (Dentes cariados, perdidos e obturados). Os idosos que tinham perdido todos os dentes (32) foram considerados "Edentados", 
e aqueles que possuíam pelo menos um dente na boca foram considerados "Dentados".

\section{Variável dependente}

A variável dependente - insatisfação com os serviços odontológicos - foi avaliada a partir da seguinte questão: "O que o Sr. (a) achou do tratamento na última consulta? (Muito bom; Bom; Regular; Ruim; Muito ruim). Para caracterização da insatisfação, as respostas "Muito bom" e "Bom" foram considerados como avaliação positiva, sendo as demais classificadas como avaliação negativa (insatisfação). Portanto, a variável dependente foi dicotomizada para avaliação da satisfação em: "sim" (satisfeito) e "não" (insatisfeito). Todas as análises e caracterizações foram feitas separadamente para idosos dentados e edentados.

\section{Variáveis independentes}

As variáveis independentes contextuais e individuais foram definidas de acordo com estudos prévios que identificaram associações de diferentes fatores com condições de saúde bucal, subjetivas e normativas, assim como características do uso de serviços odontológicos entre idosos ${ }^{8-10,17}$.

\section{Contextuais}

As variáveis contextuais consideradas na análise multinível foram: localização geográfica do município, Índice de Desenvolvimento Humano Municipal (IDHM), Índice de GINI, fluoretação das águas, presença de Centro de Especialidades Odontológicas (CEO), dentistas por mil habitantes e cobertura da equipe de saúde bucal.

A localização geográfica do município foi obtida a partir dos dados do Censo Demográfico de 2010 do IBGE. No Atlas Brasil obteve-se o IDH e índice de GINI. O IDHM é um indicador obtido pela média aritmética de três subíndices (longevidade, educação e renda), sendo classificado em: muito baixo $(\leq 0,499)$, baixo $(0,500-0,599)$, médio $(0,600-0,699)$, alto $(0,700-0,799)$ e muito alto $(\geq$ $0,800)$. Esta mesma classificação foi adotada neste estudo, porém, em função das frequências encontradas, procedeu-se à dicotomização em maior ou menor que 0,700 . O coeficiente de GINI de rendimento mede o desvio da distribuição do rendimento (ou do consumo) entre indivíduos ou famílias, internamente ao município, a partir de uma distribuição perfeitamente igual. O seu valor varia de zero (igualdade absoluta) a um (desigualdade absoluta) e foi categorizado em $\geq 0,59 /<0,59$.
A fluoretação das águas do município foi obtida a partir da Pesquisa Nacional de Saneamento Básico do IBGE, realizada em 2008 e categorizada em presente/ausente. No DATASUS foram coletadas as seguintes variáveis: média do número de cirurgiões dentistas por 1000 habitantes no município (categorizado em acima e abaixo da média 0,66); cobertura de equipes de saúde bucal na atenção primária (categorizado em acima e abaixo da meta 40\%); e presença de CEO.

\section{Individuais}

As variáveis independentes individuais foram reunidas em três grupos: socioeconômicas, atenção à saúde e condições de saúde bucal.

As condições socioeconômicas avaliadas foram: sexo, idade, raça escolaridade e renda familiar. Em relação à atenção à saúde, considerou-se o tipo de serviço odontológico utilizado e o motivo do uso.

Nas condições de saúde bucal foram incluídas variáveis referentes ao uso de prótese dentária, dor de dente nos últimos seis meses, satisfação com dentes e boca, auto percepção da necessidade de tratamento e impacto da saúde bucal na qualidade de vida. Tal impacto foi mensurado a partir do instrumento Oral Impacts on Daily Performance (OIDP) ${ }^{18}$, que avalia o impacto das condições bucais na habilidade do indivíduo em desenvolver atividades diárias, sendo considerados nove itens. Para avaliação do impacto geral na qualidade de vida foi realizada a junção dos nove itens, sendo as opções de resposta: "não" (ausência de impacto em todos os itens) e "sim" (impacto em pelo menos um item).

\section{Análise estatística e considerações éticas}

Inicialmente, todas as variáveis foram descritas quanto aos seus valores absolutos (n) e porcentagem (\%), considerando a correção pelo efeito de desenho, utilizando o software Statistical Package for the Social Sciences ${ }^{\circledR}$ (SPSS 19.0). Posteriormente, foi realizada análise bivariada para todas as variáveis independentes, sendo consideradas na análise multinível as variáveis individuais com valor de p menor que 0,20 , estimou-se também o odds ratio e intervalo de confiança (IC95\%).

$\mathrm{Na}$ análise estatística multinível, inicialmente foram consideradas as variáveis contextuais e, em um segundo momento, as individuais, utilizando o programa STATA ${ }^{\circledR}$ 14.0. Todas as variáveis contextuais foram testadas nos modelos multiníveis. 
A análise multinível utilizou o modelo de efeitos fixos (modelo de intercepto) para rodar a relação entre o desfecho (insatisfação serviços) e as variáveis explicativas de primeiro nível (cidades participantes), para permitir a identificação das variáveis contextuais significativas ( $\mathrm{p} \leq$ $0,05)$ que seriam consideradas juntamente com as de segundo nível (variáveis individuais). No modelo 1 foram consideradas apenas as variáveis contextuais ajustadas juntamente com as variáveis individuais com $p$ menor que 0,20 na análise bivariada. As variáveis com valor de $\mathrm{p}$ menor que 0,05 no modelo 1 foram consideradas para construção do modelo final ajustado (Modelo 2), sendo mantidas no modelo apenas variáveis com nível de significância de até 5\%.

O levantamento epidemiológico foi conduzido respeitando-se os princípios éticos da Resolução do Conselho Nacional de Saúde nº196/96, sendo aprovado e registrado pela Comissão Nacional de Ética em Pesquisa (Conep).

\section{Resultados}

Um total de 1989 idosos atenderam aos critérios de inclusão e foram incluídos nesse estudo. Destes, 1566 eram dentados e 423 edentados. $\mathrm{Na}$ avaliação dos serviços odontológicos, 11,2\% dos idosos dentados e $22,1 \%$ dos edentados estavam insatisfeitos. A amostra foi composta em sua maioria pelo sexo feminino. Identificou-se que para ambas as amostras (dentados e edentados), a maioria era composta por idosos que residiam em municípios que não possuíam CEO, com a cobertura de equipes de saúde bucal menor que $40 \%$ e IDH maior ou igual a 0,7 . Além disso, vale destacar que para ambos os grupos de idosos (dentados e edentados) houve o predomínio do uso de serviço odontológico particular, de convênio ou filantrópico. Quanto à condição de saúde bucal, a maioria dos idosos usava prótese, sentiu dor de dente nos últimos 6 meses, estava satisfeito com seus dentes e sua boca, e não houve impacto da sua saúde bucal na qualidade de vida. Em contrapartida, somente a maioria dos idosos edentados percebeu a necessidade de tratamento (Tabela 1).

$\mathrm{Na}$ análise bivariada, identificou-se associação $(p \leq 0,20)$ com variáveis contextuais, socioeconômicas, atenção à saúde e condições de saúde bucal. Já entre os edentados, apenas variáveis referentes à atenção à saúde e condições de saúde bucal apontaram associação ( $\mathrm{p} \leq 0,20)$ (Tabela 2).
Na tabela 3 foi construída a analise multinível, considerando primeiramente o modelo vazio a partir da variável dependente, onde em seguida foram incluídas as variáveis contextuais (agregação por município). Ressalta-se que após o ajuste do modelo, entre os idosos edentados, somente a localização do município esteve associada ao desfecho (dependente) $(\mathrm{p} \leq 0,05)$. Por outro lado, para os idosos dentados, somente a fluoretação das águas não esteve associada ao desfecho (Tabela 3).

$\mathrm{Na}$ análise multinível entre idosos dentados (Modelo 1), considerou-se as variáveis contextuais associadas ao desfecho no modelo multinível ajustado e as individuais identificadas na bivariada. A partir do modelo ajustado (Modelo 2) (p $\leq 0,05)$ identificou-se que variáveis contextuais (localização do município, IDH, Índice de GINI, presença de CEO e cobertura das equipes de saúde bucal), individuais pertencentes à atenção à saúde (motivo do uso) e as variáveis relativas às condições de saúde bucal dos idosos, com exceção do uso de prótese e impacto na qualidade de vida, permaneceram associadas à insatisfação com os serviços odontológicos (Tabela 4).

No modelo multinível, entre idosos edentados apenas o motivo do uso e a satisfação com dentes e boca permaneceram associados ( $\mathrm{p} \leq$ $0,05)$ à insatisfação com os serviços odontológicos (Modelo 2) (Tabela 5).

\section{Discussão}

Identificar a prevalência e os fatores associados à insatisfação dos usuários em relação aos serviços de saúde tem grande importância na avaliação, planejamento e gestão desses serviços. No presente estudo, identificou-se que uma parcela considerável de idosos relatou estar insatisfeita com os serviços odontológicos, sendo esta avaliação negativa maior entre idosos edentados. Estudos prévios que avaliaram a insatisfação entre idosos brasileiros com os serviços odontológicos, sem considerar o edentulismo, identificaram prevalências entre $8,7 \%$ a $14,4 \%^{8-10}$. A prevalência de insatisfação encontrada no presente estudo foi semelhante a identificada na Noruega ${ }^{19}$; e inferior $(8 \%)$ à registrada no Reino Unido ${ }^{20}$. Porém, diferenças metodológicas entre os estudos devem ser consideradas como por exemplo, critérios de inclusão, instrumentos utilizados para a medição da insatisfação e a condição socioeconômica dos países. A maior insatisfação entre idosos edentados pode ser explicada pelo fato de que a ausência 
Tabela 1. Análise descritiva das variáveis contextuais e individuais entre idosos brasileiros dentados e edentados. 2010.

\begin{tabular}{|c|c|c|c|c|}
\hline \multirow[t]{2}{*}{ Variáveis } & \multicolumn{2}{|c|}{ Dentados } & \multicolumn{2}{|c|}{ Edentados } \\
\hline & $\mathbf{N}$ & $\%$ & $\mathbf{N}$ & $\%$ \\
\hline \multicolumn{5}{|l|}{ Satisfação com os serviços odontológicos } \\
\hline Sim (Muito bom/ bom) & 1360 & 88,8 & 362 & 77,2 \\
\hline Não (Regular/ Ruim/ Péssimo) & 206 & 11,2 & 61 & 22,1 \\
\hline \multicolumn{5}{|l|}{ Contextuais } \\
\hline \multicolumn{5}{|l|}{ Localização geográfica do município } \\
\hline Capital & 1350 & 65,1 & 329 & 71,6 \\
\hline Interior & 216 & 34,9 & 94 & 28,4 \\
\hline \multicolumn{5}{|l|}{ IDH } \\
\hline Maior ou igual a 0,7 & 1422 & 90,0 & 354 & 78,8 \\
\hline Menor que 0,7 & 144 & 10,0 & 69 & 21,2 \\
\hline \multicolumn{5}{|l|}{ Índice GINI } \\
\hline Menor ou igual a 0,59 & 421 & 35,6 & 148 & 27,6 \\
\hline Maior que 0,59 & 1145 & 64,4 & 275 & 72,4 \\
\hline \multicolumn{5}{|l|}{ Fluoretação das águas } \\
\hline Sim & 1279 & 90,2 & 324 & 83,3 \\
\hline Não & 287 & 9,8 & 99 & 16,7 \\
\hline \multicolumn{5}{|l|}{ Presença de CEO* } \\
\hline Sim & 592 & 42,3 & 163 & 48,4 \\
\hline Não & 926 & 57,7 & 242 & 51,6 \\
\hline \multicolumn{5}{|l|}{ Dentistas por mil habitantes } \\
\hline Acima da média $(0,66)$ & 1212 & 65,7 & 298 & 58,1 \\
\hline Abaixo da Média $(0,66)$ & 354 & 34,3 & 125 & 41,9 \\
\hline \multicolumn{5}{|l|}{ Cobertura Equipe de saúde Bucal } \\
\hline Maior ou igual a $40 \%$ & 369 & 36,5 & 130 & 47,1 \\
\hline Menor que $40 \%$ & 1197 & 63,5 & 293 & 52,9 \\
\hline \multicolumn{5}{|l|}{ Individuais } \\
\hline \multicolumn{5}{|l|}{ Socioeconômicas } \\
\hline \multicolumn{5}{|l|}{ Sexo } \\
\hline Feminino & 936 & 62,0 & 286 & 57,0 \\
\hline Masculino & 630 & 38,0 & 137 & 43,0 \\
\hline \multicolumn{5}{|l|}{ Idade } \\
\hline 64-69 anos & 995 & 56,0 & 229 & 50,9 \\
\hline 70-74anos & 571 & 43,8 & 194 & 49,1 \\
\hline \multicolumn{5}{|l|}{ Raça } \\
\hline Branco & 879 & 66,1 & 219 & 52,4 \\
\hline Negro/Amarelo/Pardo/Indígena & 687 & 33,9 & 204 & 47,6 \\
\hline \multicolumn{5}{|l|}{ Escolaridade $^{\star}$} \\
\hline Acima de 5 anos & 1006 & 59,9 & 164 & 40,6 \\
\hline 1 a 4 anos & 356 & 29,6 & 162 & 38,9 \\
\hline Analfabeto & 193 & 10,5 & 89 & 20,6 \\
\hline \multicolumn{5}{|l|}{ Renda Familiar (pela mediana)* } \\
\hline Maior que 1500 reais & 821 & 50,8 & 127 & 26,2 \\
\hline Menor ou igual 1500 reais & 676 & 49,2 & 278 & 73,8 \\
\hline
\end{tabular}

de dentes pode impactar negativamente na vida de idosos, alterando sua percepção e importância que se dá à saúde bucal ${ }^{14,15}$.
Um dado interessante é o fato de que a baixa prevalência da insatisfação tem sido reportada como efeito da "elevação" das taxas de satisfação, 
Tabela 1. Análise descritiva das variáveis contextuais e individuais entre idosos brasileiros dentados e edentados. 2010.

\begin{tabular}{|c|c|c|c|c|}
\hline \multirow[t]{2}{*}{ Variáveis } & \multicolumn{2}{|c|}{ Dentados } & \multicolumn{2}{|c|}{ Edentados } \\
\hline & $\mathbf{N}$ & $\%$ & $\mathbf{N}$ & $\%$ \\
\hline \multicolumn{5}{|l|}{ Atenção à Saúde } \\
\hline \multicolumn{5}{|l|}{ Tipo de serviço odontológico utilizado } \\
\hline SUS & 421 & 19,7 & 120 & 23,4 \\
\hline Outros (particular, convênio ou filantrópico) & 1145 & 80,3 & 303 & 76,6 \\
\hline \multicolumn{5}{|l|}{ Motivo uso dos serviços odontológicos ${ }^{\star}$} \\
\hline Rotina & 398 & 26,6 & 71 & 9,5 \\
\hline Tratamento & 1166 & 73,4 & 349 & 90,5 \\
\hline \multicolumn{5}{|l|}{ Condições de saúde bucal } \\
\hline \multicolumn{5}{|l|}{ Uso de prótese ${ }^{\star}$} \\
\hline Não & 463 & 26,0 & 31 & 5,9 \\
\hline Sim & 1102 & 74,0 & 390 & 94,1 \\
\hline \multicolumn{5}{|l|}{ Dor de dentes nos últimos 6 meses $^{*}$} \\
\hline Não & 1248 & 80,8 & 337 & 88,4 \\
\hline Sim & 314 & 19,2 & 30 & 11,6 \\
\hline \multicolumn{5}{|l|}{ Satisfação dentes e boca* } \\
\hline Satisfeito & 833 & 53,8 & 273 & 53,0 \\
\hline Insatisfeito & 730 & 46,2 & 148 & 47,0 \\
\hline \multicolumn{5}{|l|}{ Auto percepção necessidade tratamento* } \\
\hline Não & 552 & 41,1 & 273 & 64,6 \\
\hline Sim & 1002 & 58,9 & 144 & 35,4 \\
\hline \multicolumn{5}{|l|}{ Impacto da saúde bucal na qualidade de vida ${ }^{*}$} \\
\hline Não & 866 & 55,5 & 252 & 51,0 \\
\hline Sim & 700 & 44,5 & 171 & 49,0 \\
\hline
\end{tabular}

relatada mesmo quando as expectativas sobre os serviços são negativas ${ }^{21}$. Em adição, salienta-se a resiliência de idosos, que podem se conformar ou aceitar algumas condições mesmo quando estas são negativas ${ }^{22}$. No entanto, sendo a avaliação do usuário uma das medidas de qualidade do serviço, a baixa insatisfação pode indicar a resolutividade do mesmo.

Entre idosos dentados, identificou-se a insatisfação com os serviços odontológicos associada às variáveis contextuais dos municípios e individuais. Em relação às variáveis contextuais, a chance de insatisfação foi maior entre os idosos residentes no interior, em municípios com índice de GINI maior que 0,59 e com uma menor cobertura de equipes de saúde bucal na atenção básica. Uma menor chance de insatisfação foi observada entre idosos residentes em municípios com IDH menor que 0,7 e que não possuíam CEO. Sabe-se que os indivíduos interagem com o contexto social a que pertencem, são influenciados por ele e, ao mesmo tempo, também influen- ciam o contexto em que estão inseridos ${ }^{23}$. Usuários residentes em interiores que possuem menor estrutura e menor oferta de serviços de saúde, incluindo a atenção primária, e, possivelmente, maior desigualdade de renda, possuem maior dificuldade de acesso aos serviços odontológicos, podendo acarretar em uma maior insatisfação ${ }^{24}$. Em relação ao IDH, um de seus componentes diz respeito à escolaridade. Desta forma, pessoas com níveis mais elevados de escolaridade poderiam avaliar o atendimento odontológico mais negativamente $e^{9,21}$, o que demonstra uma maior exigência por parte destes ${ }^{9,25}$. Destaca-se ainda que, a ausência do CEO em municípios acarreta em problemas no acesso de idosos à serviços odontológicos especializados, podendo comprometer as condições de saúde bucal e levar a uma maior insatisfação com a assistência odontológica. Os fatores contextuais têm sido associados a agravos bucais, tais como a cárie dentária ${ }^{26}$ e dor de dente ${ }^{27}$, assim como ao uso de serviços odontológicos ${ }^{28}$. No entanto, a associação da insatis- 
Tabela 2. Análise bivariada entre a insatisfação com os serviços odontológicos e as variáveis contextuais e individuais entre idosos brasileiros dentados e edentados. 2010.

\begin{tabular}{|c|c|c|c|c|c|c|}
\hline \multirow{2}{*}{$\begin{array}{c}\text { Variáveis } \\
\text { Contextuais }\end{array}$} & \multicolumn{3}{|c|}{ Dentados } & \multicolumn{3}{|c|}{ Edentados } \\
\hline & $\%$ & OR (IC95\%) & p & $\%$ & OR (IC95\%) & p \\
\hline \multicolumn{7}{|l|}{ Localização geográfica do município } \\
\hline Capital & 13,3 & & & 13,4 & & \\
\hline Interior & 12,0 & $0,88(0,57-1,38)$ & & 18,1 & $1,43(0,77-2,64)$ & \\
\hline \multicolumn{7}{|l|}{ IDH } \\
\hline Maior ou igual a 0,7 & 13,6 & & & 15,0 & & \\
\hline Menor que 0,7 & 8,3 & $0,57(0,31-1,05)$ & & 11,6 & $0,74(0,33-1,64)$ & \\
\hline \multicolumn{7}{|l|}{ Índice GINI } \\
\hline Menor ou igual a 0,59 & 9,5 & & & 13,5 & & \\
\hline Maior que 0,59 & 14,5 & $1,61(1,12-2,32)$ & & 14,9 & $1,12(0,63-1,99)$ & \\
\hline \multicolumn{7}{|l|}{ Fluoretação das águas } \\
\hline Sim & 13,4 & & & 14,2 & & \\
\hline Não & 12,2 & $0,90(0,61-1,32)$ & & 15,2 & $1,07(0,57-2,03)$ & \\
\hline \multicolumn{7}{|l|}{ Presença de CEO } \\
\hline $\operatorname{Sim}$ & 16,9 & & & 15,3 & & \\
\hline Não & 10,9 & $0,60(0,44-0,81)$ & & 14,5 & $0,93(0,53-1,62)$ & \\
\hline \multicolumn{7}{|l|}{ Dentistas por mil habitantes } \\
\hline Acima da média $(0,66)$ & 12,5 & & & 13,4 & & \\
\hline Abaixo da Média $(0,66)$ & 15,5 & $0,13(0,92-1,80)$ & & 16,8 & $1,30(0,73-2,31)$ & \\
\hline \multicolumn{7}{|l|}{ Cobertura Equipe de saúde Bucal } \\
\hline Maior ou igual a $40 \%$ & 9,8 & & & 14,6 & & \\
\hline Menor que $40 \%$ & 14,2 & $1,53(1,04-2,24)$ & & 14,3 & $0,97(0,54-1,75)$ & \\
\hline \multicolumn{7}{|l|}{ Individuais } \\
\hline \multicolumn{7}{|l|}{ Socioeconômicas } \\
\hline \multicolumn{7}{|l|}{ Sexo } \\
\hline Feminino & 12,0 & & & 14,3 & & \\
\hline Masculino & 14,9 & $1,29(0,96-1,73)$ & 0,090 & 14,6 & $1,02(0,57-1,82)$ & 0,943 \\
\hline \multicolumn{7}{|l|}{ Idade } \\
\hline 64-69 anos & 12,9 & & & 16,2 & & \\
\hline 70-74anos & 13,7 & $1,07(0,79-1,45)$ & 0,654 & 12,4 & $0,73(0,42-1,27)$ & 0,271 \\
\hline \multicolumn{7}{|l|}{ Raça } \\
\hline Branco & 11,1 & & & 15,5 & & \\
\hline Negro/Amarelo/Pardo/Indígena & 15,7 & $1,48(1,10-1,99)$ & 0,008 & 13,2 & $0,83(0,48-1,43)$ & 0,503 \\
\hline \multicolumn{7}{|l|}{ Escolaridade } \\
\hline Acima de 5 anos & 12,2 & & & 15,9 & & \\
\hline 1 a 4 anos & 14,0 & $1,17(0,82-1,67)$ & 0,375 & 13,0 & $0,79(0,42-1,47)$ & 0,458 \\
\hline Analfabeto & 16,1 & $1,37(0,89-2,10)$ & 0,146 & 14,6 & $0,90(0,44-1,89)$ & 0,793 \\
\hline \multicolumn{7}{|l|}{ Renda Familiar (pela mediana) } \\
\hline Maior que 1500 reais & 11,4 & & & 14,2 & & \\
\hline Menor ou igual 1500 reais & 14,9 & $1,35(1,00-1,83)$ & 0,046 & 15,1 & $1,07(0,59-1,95)$ & 0,806 \\
\hline \multicolumn{7}{|l|}{ Atenção a saúde } \\
\hline \multicolumn{7}{|l|}{ Tipo de serviço odontológico utilizado } \\
\hline $\begin{array}{l}\text { Outros (particular, convênio ou } \\
\text { filantrópico) }\end{array}$ & 12,4 & & & 15,5 & & \\
\hline SUS & 15,2 & $1,26(0,92-1,74)$ & 0,147 & 11,7 & $0,71(0,38-1,36)$ & 0,312 \\
\hline \multicolumn{7}{|l|}{ Motivo do uso } \\
\hline Rotina & 7,3 & & & 5,6 & & \\
\hline Tratamento & 15,2 & $2,27(1,51-3,43)$ & $<0,001$ & 16,3 & $3,27(1,14-9,32)$ & 0,027 \\
\hline
\end{tabular}


Tabela 2. Análise bivariada entre a insatisfação com os serviços odontológicos e as variáveis contextuais e individuais entre idosos brasileiros dentados e edentados. 2010.

\begin{tabular}{|c|c|c|c|c|c|c|}
\hline \multirow{2}{*}{$\begin{array}{c}\text { Variáveis } \\
\text { Contextuais }\end{array}$} & \multicolumn{3}{|c|}{ Dentados } & \multicolumn{3}{|c|}{ Edentados } \\
\hline & $\%$ & OR (IC95\%) & $\mathbf{p}$ & $\%$ & OR (IC95\%) & p \\
\hline \multicolumn{7}{|l|}{ Condições de saúde bucal } \\
\hline \multicolumn{7}{|l|}{ Uso de prótese } \\
\hline Não & 14,7 & & & 12,9 & & \\
\hline Sim & 12,5 & $0,83(0,60-1,13)$ & 0,200 & 14,6 & $1,15(0,39-4,42)$ & 0,795 \\
\hline \multicolumn{7}{|c|}{ Dor de dentes nos últimos 6 meses } \\
\hline Não & 10,4 & & & 13,6 & & \\
\hline Sim & 23,9 & $2,69(1,96-3,70)$ & $<0,001$ & 20,0 & $1,58(0,61-4,07)$ & 0,343 \\
\hline \multicolumn{7}{|l|}{ Satisfação dentes e boca } \\
\hline Satisfeito & 6,1 & & & 8,1 & & \\
\hline Insatisfeito & 21,2 & $4,13(2,95-5,77)$ & $<0,001$ & 26,4 & $4,08(2,31-7,21)$ & $<0,001$ \\
\hline \multicolumn{7}{|c|}{ Auto percepção necessidade tratamento } \\
\hline Não & 5,4 & & & 12,5 & & \\
\hline $\operatorname{Sim}$ & 17,2 & $3,60(2,41-5,39)$ & $<0,001$ & 18,8 & $1,62(0,93-2,81)$ & 0,086 \\
\hline \multicolumn{7}{|c|}{ Impacto da saúde bucal na qualidade de vida } \\
\hline Não & 9,8 & & & 11,5 & & \\
\hline $\operatorname{Sim}$ & 17,3 & $1,92(1,42-2,58)$ & $<0,001$ & 18,7 & $1,77(1,02-3,05)$ & 0,040 \\
\hline
\end{tabular}

Tabela 3. Modelo múltiplo multinível da associação entre a insatisfação com os serviços odontológicos e as variáveis contextuais entre idosos brasileiros dentados e edentados, 2010.

\begin{tabular}{|c|c|c|c|c|c|c|}
\hline \multirow[t]{2}{*}{ Variáveis } & \multicolumn{3}{|c|}{ Dentados } & \multicolumn{3}{|c|}{ Edentados } \\
\hline & OR & IC95\% & $\mathbf{p}$ & OR & IC95\% & p \\
\hline \multicolumn{7}{|c|}{ Localização geográfica do município } \\
\hline Capital & 1 & & & 1 & & \\
\hline Interior & $2,14^{*}$ & $1,03-4,46$ & 0,040 & 2,84 & $1,00-8,09$ & 0,049 \\
\hline \multicolumn{7}{|l|}{$\mathrm{IDH}$} \\
\hline Maior ou igual a 0,7 & 1 & & & 1 & & \\
\hline Menor que 0,7 & $0,33^{*}$ & $0,12-0,88$ & 0,028 & 0,40 & $0,12-1,28$ & 0,124 \\
\hline \multicolumn{7}{|l|}{ Índice GINI } \\
\hline Menor ou igual a 0,59 & 1 & & & 1 & & \\
\hline Maior que 0,59 & $1,94^{*}$ & $1,13-3,32$ & 0,016 & 1,78 & $0,80-3,96$ & 0,152 \\
\hline \multicolumn{7}{|l|}{ Fluoretação das águas } \\
\hline Sim & 1 & & & 1 & & \\
\hline Não & 0,96 & $0,60-1,52$ & 0,864 & 0,98 & $0,49-1,95$ & 0,962 \\
\hline \multicolumn{7}{|l|}{ Presença de CEO } \\
\hline Sim & 1 & & & 1 & & \\
\hline Não & $0,64^{*}$ & $0,45-0,91$ & 0,013 & 0,97 & $0,55-1,72$ & 0,939 \\
\hline \multicolumn{7}{|l|}{ Dentistas por mil habitantes } \\
\hline Acima da média $(0,66)$ & 1 & & & 1 & & \\
\hline Abaixo da Média $(0,66)$ & $1,55^{\star}$ & $1,02-2,36$ & 0,039 & 1,47 & $0,76-2,86$ & 0,245 \\
\hline \multicolumn{7}{|c|}{ Cobertura Equipe de saúde Bucal } \\
\hline Maior ou igual a 40\% & 1 & & & 1 & & \\
\hline Menor que $40 \%$ & $1,67^{\star}$ & $1,07-2,61$ & 0,023 & 1,13 & $0,59-2,18$ & 0,696 \\
\hline
\end{tabular}

fação com os serviços odontológicos e variáveis contextuais entre idosos parece ser pioneira na literatura nacional.
Entre os idosos dentados, identificou-se também a associação da insatisfação com características individuais. Foi constatada uma maior 
Tabela 4. Modelo múltiplo mutinível da associação entre a insatisfação dos serviços odontológico com as variáveis contextuais e individuais entre idosos dentados. 2010.

\begin{tabular}{|c|c|c|c|c|c|c|}
\hline \multirow{2}{*}{ Contextuais } & \multicolumn{3}{|c|}{ Modelo 1} & \multicolumn{3}{|c|}{ Modelo 2} \\
\hline & OR & IC95\% & $\mathbf{p}$ & OR & IC95\% & $\mathbf{P}$ \\
\hline \multicolumn{7}{|l|}{ Localização geográfica do município } \\
\hline Capital & 1 & & & 1 & & \\
\hline Interior & 2.24 & $1,03-4,86$ & 0,040 & 2,72 & $1,31-5,64$ & 0,007 \\
\hline \multicolumn{7}{|l|}{ IDH } \\
\hline Maior ou igual a 0,7 & 1 & & & 1 & & \\
\hline Menor que 0,7 & 0,23 & $0,07-0,70$ & 0,010 & 0,29 & $0,10-0,78$ & 0,015 \\
\hline \multicolumn{7}{|l|}{ Índice GINI } \\
\hline Menor ou igual a 0,59 & 1 & & & 1 & & \\
\hline Maior que 0,59 & 1,60 & $0,94-2,73$ & 0,080 & 1,92 & $1,16-3.17$ & 0,011 \\
\hline \multicolumn{7}{|l|}{ Presença de CEO } \\
\hline Sim & 1 & & & 1 & & \\
\hline Não & 0,56 & $0,39-0,81$ & 0,002 & 0,58 & $0,41-0,80$ & 0,001 \\
\hline \multicolumn{7}{|l|}{ Dentistas por mil habitantes } \\
\hline Acima da média $(0,66)$ & 1 & & & -- & & \\
\hline Abaixo da Média $(0,66)$ & 1,30 & $0,83-2,02$ & 0,242 & & & \\
\hline \multicolumn{7}{|l|}{ Cobertura Equipe de saúde Bucal } \\
\hline Maior ou igual a $40 \%$ & 1 & & & 1 & & \\
\hline Menor que $40 \%$ & 1,95 & $1,20-3,16$ & 0,006 & 1,76 & $1,13-2,74$ & 0,012 \\
\hline \multicolumn{7}{|l|}{ Individuais } \\
\hline \multicolumn{7}{|l|}{ Socioeconômicas } \\
\hline \multicolumn{7}{|l|}{ Sexo } \\
\hline Feminino & 1 & & & -- & & \\
\hline Masculino & 1,26 & $0,89-1,76$ & 0,179 & & & \\
\hline \multicolumn{7}{|l|}{ Raça } \\
\hline Branco & 1 & & & -- & & \\
\hline Negro/Amarelo/Pardo/Indígena & 1,32 & $0,91-1,90$ & 0,132 & & & \\
\hline \multicolumn{7}{|l|}{ Escolaridade } \\
\hline Acima de 5 anos & 1 & & & -- & & \\
\hline 1 a 4 anos & 0,81 & $0,52-1,26$ & 0,359 & & & \\
\hline \multicolumn{7}{|l|}{ Analfabeto } \\
\hline \multicolumn{7}{|l|}{ Renda Familiar (pela mediana) } \\
\hline Maior que 1500 reais & 1 & & & -- & & \\
\hline Menor ou igual 1500 reais & 1,04 & $0,70-1,54$ & 0,816 & & & \\
\hline \multicolumn{7}{|l|}{ Atenção a saúde } \\
\hline \multicolumn{7}{|l|}{ Tipo de serviço odontológico utilizado } \\
\hline Outros (particular, convênio ou filantrópico) & 1 & & & -- & & \\
\hline SUS & 0,93 & $0,61-1,41$ & 0,740 & & & \\
\hline \multicolumn{7}{|l|}{ Motivo do uso } \\
\hline Rotina & 1 & & & 1 & & \\
\hline Tratamento & 1,77 & $1,07-2,92$ & 0,025 & 1,63 & $1,03-2,57$ & 0,035 \\
\hline \multicolumn{7}{|l|}{ Condições de saúde bucal } \\
\hline Uso de prótese & & & & & & \\
\hline Não & 1 & & & -- & & \\
\hline Sim & 1,15 & $0,78-1,69$ & 0,460 & & & \\
\hline Dor de dentes nos últimos 6 meses & & & & & & \\
\hline Não & 1 & & & 1 & & \\
\hline Sim & 2,18 & $1,49-3,20$ & $<0,001$ & 2,02 & $1,42-2,88$ & $<0,001$ \\
\hline Satisfação dentes e boca & & & & & & \\
\hline Satisfeito & 1 & & & 1 & & \\
\hline Insatisfeito & 2,61 & $1,76-3,87$ & $<0,001$ & 2,65 & $1,84-3,81$ & $<0,001$ \\
\hline
\end{tabular}


Tabela 4. Modelo múltiplo mutinível da associação entre a insatisfação dos serviços odontológico com as variáveis contextuais e individuais entre idosos dentados. 2010.

\begin{tabular}{|c|c|c|c|c|c|c|}
\hline \multirow{2}{*}{ Contextuais } & \multicolumn{3}{|c|}{ Modelo 1} & \multicolumn{3}{|c|}{ Modelo 2} \\
\hline & OR & IC95\% & $\mathbf{p}$ & OR & IC95\% & $\mathbf{P}$ \\
\hline \multicolumn{7}{|c|}{ Auto percepção necessidade tratamento } \\
\hline Não & 1 & & & 1 & & \\
\hline Sim & 2,10 & $1,31-3,37$ & 0,002 & 2,13 & $1,36-3,33$ & 0,001 \\
\hline \multicolumn{7}{|c|}{ Impacto da saúde bucal na qualidade de vida } \\
\hline Não & 1 & & & -- & & \\
\hline Sim & 1,08 & $0,75-1,56$ & 0,660 & & & \\
\hline
\end{tabular}

Tabela 5. Modelo múltiplo mutinível da associação entre a insatisfação dos serviços odontológico com as variáveis contextuais e individuais entre idosos edentados. 2010.

\begin{tabular}{|c|c|c|c|c|c|c|}
\hline \multirow{2}{*}{ Variáveis } & \multicolumn{3}{|c|}{ Modelo 1} & \multicolumn{3}{|c|}{ Modelo 2} \\
\hline & OR & IC95\% & $\mathbf{p}$ & OR & IC95\% & $\mathbf{p}$ \\
\hline \multicolumn{7}{|l|}{ Motivo do uso } \\
\hline Rotina & 1 & & & 1 & & \\
\hline Tratamento & 2,92 & $1,00-8,51$ & 0,049 & 2,87 & $0,99-8,33$ & 0,050 \\
\hline \multicolumn{7}{|l|}{ Condições de saúde bucal } \\
\hline \multicolumn{7}{|l|}{ Satisfação dentes e boca } \\
\hline Satisfeito & 1 & & & 1 & & \\
\hline Insatisfeito & 3,97 & $2,08-7,57$ & $<0,001$ & 3,85 & $2,17-6,83$ & $<0,001$ \\
\hline \multicolumn{7}{|c|}{ Auto percepção necessidade tratamento } \\
\hline Não & 1 & & & -- & & \\
\hline Sim & 0,94 & $0,51-1,75$ & 0,867 & & & \\
\hline \multicolumn{7}{|c|}{ Impacto da saúde bucal na qualidade de vida } \\
\hline Não & 1 & & & -- & & \\
\hline Sim & 1,01 & $0,55-1,87$ & 0,958 & & & \\
\hline
\end{tabular}

chance de insatisfação entre idosos dentados que usaram serviços odontológicos para tratamento, com dor de dentes, que estavam insatisfeitos com seus dentes e boca e perceberam a necessidade de tratamento. Uma maior insatisfação entre idosos com dor de dente e insatisfeitos com suas condições de saúde bucal, foi identificada também em estudo prévio considerando dados do Levantamento Nacional de Saúde bucal realizado em 2002/2003 ${ }^{9}$.

Tem-se identificado associação entre a necessidade de tratamento odontológico e o uso de serviços por idosos brasileiros ${ }^{29}$. Estudo de coorte realizado entre idosos do Sul do Brasil identificou que a satisfação com o tratamento com próteses dentárias esteve associada à qualidade da prótese e à execução de funções bucais ${ }^{30}$. Portanto, a qualidade do tratamento executado e a resolução do problema pode influenciar na insatisfação com os serviços entre aqueles que o utilizam para realização desse tratamento. Além disso, uma associação entre a insatisfação com a assistência odontológica e a percepção do usuário em achar que o profissional não possui habilidades adequada, já foi relatado na literatura ${ }^{10}$.

Em relação aos idosos edentados, foi identificada associação entre a insatisfação com os serviços e as variáveis individuais. Constatou-se entre idosos edentados uma maior insatisfação entre aqueles que utilizaram serviço odontológico para tratamento e estavam insatisfeitos com sua condição de saúde bucal. Assim como para os idosos dentados, o uso para tratamento pode levar à não resolução do problema bucal, avaliação negativa da qualidade do serviço ou dificuldades no acesso, o que resulta em insatisfação com os serviços. Estudo prévio conduzido entre idosos reabilitados com próteses totais por um CEO em um 
município no nordeste do Brasil, constatou que as próteses satisfizeram os usuários do ponto de vista estético, mas não pela sua funcionalidade ${ }^{31}$. Em relação à satisfação com a saúde bucal, esta pode ser considerada um processo complexo e depende da relação paciente/dentista que resulta de experiências com o tratamento anteriormente vivido ${ }^{32}$. Portanto, a insatisfação com a saúde bucal pode estar relacionada a tratamentos inadequados realizados e dificuldade de acesso para tratamento de enfermidades bucais ou reabilitação oral, o que pode acarretar na insatisfação com o serviço.

Ressalta-se que, o processo de avaliação de serviços de saúde é dinâmico, sendo resultado da experiência vivida e perspectivas do paciente que podem ser facilmente alteradas a depender do seu estado emocional. Assim, pode ser necessária a avaliação de outros aspectos relacionados à estrutura do serviço e à relação profissional-paciente com intuito de compreender o que levou à insatisfação. Tendo em vista que apenas uma questão foi avaliada, esta pode ser considerada uma limitação do presente estudo. Apesar disto, os resultados aqui identificados podem nortear o aprimoramento de políticas de saúde bucal com intuito de atender apropriadamente às necessidades da população idosa e melhor a qualidade da assistência fornecida. Além disso, gestores poderiam ser conscientizados de que a presença de dentes na boca pode resultar na necessidade dos serviços para diferentes tratamentos e com diferentes expectativas a serem atendidas, consequentemente, associando-se a diferentes fatores. Desta forma, deve-se considerar também as políticas vigentes e o ambiente em que os indivíduos estão inseridos.

Portanto, os resultados demonstraram que uma parcela considerável de idosos brasileiros avaliaram negativamente o serviço odontológico utilizado. Em adição, nota-se que a presença de dentes na cavidade bucal parece influenciar nesta avaliação e em seus possíveis fatores associados, uma vez que as variáveis contextuais se mantiveram associadas apenas entre os idosos dentados.

\section{Colaboradores}

JGS Souza, AMEBL Martins e RF Oliveira participaram da concepção da ideia, organização e análise dos dados e redação do artigo. BEC Oliveira, AA Sampaio, MS Noronha e CV Lima participaram da análise dos dados e redação do artigo. EF Ferreira participou da revisão crítica.

\section{Agradecimentos}

Agradecimentos ao fomento da Fundação de Amparo à Pesquisa do Estado de Minas GeraisFAPEMIG. 


\section{Referências}

1. Serapioni M. Avaliação de qualidade em saúde: a contribuição da sociologia da saúde para superação da polarização entre visão dos usuários e a perspectiva dos profissionais da saúde. Saúde em Debate 1999; 23(53):81-92.

2. Donabedian A. The quality of care: how can it be assessed? JAMA 1988; 260(12):1743-1748.

3. Bottan ER, Sperb RAL, Telles PS, Uriarte Neto M. Avaliação de serviços odontológicos: a visão dos pacientes. Rev ABENO 2006; 6(2):128-133.

4. Martins AMEBL, Barreto SM, Pordeus IA. Uso de serviços odontológicos entre idosos brasileiros. $\mathrm{Rev} \mathrm{Pa}$ nam Salud Publica 2007; 22(5):308-316.

5. Gouveia GC, Souza WV, Luna CF, Souza-Júnior PRB, Szwarcwald CL. Satisfação dos usuários do sistema de saúde brasileiro: fatores associados e diferenças regionais. Rev Bras Epidemiol. 2009; 12(3):281-296.

6. Bleich SN, Ozaltin E, Murray CJL. How does satisfaction with the health-care system relate to patient experience? Bull World Health Organ 2009; 87(4):271-278.

7. Benazzi LEB, Figueiredo ACL, Bassani DG. Avaliação do usuário sobre o atendimento oftalmológico oferecido pelo SUS em um centro urbano no sul do Brasil. Cien Saude Colet 2010; 15(3):861-868.

8. Rodrigues CAQ, Silva PLV, Caldeira AP, Pordeus IA, Ferreira RC, Martins AMEBL. Fatores associados à satisfação com serviços odontológicos entre idosos. Rev Saude Publica 2012; 46(6):1039-1050.

9. Martins AMEBL, Jardim LA, Souza JGS, Rodrigues CAQ, Ferreira RC, Pordeus IA. Is the negative evaluation of dental services among the Brazilian elderly population associated with the type of service? Rev Bras Epidemiol 2014; 17(4):71-90.

10. Martins AMEBL, Ferreira RC, Santos-Neto PE, Carreiro DL, Souza JGS, Ferreira EF. Insatisfação dos usuários com a assistência odontológica: estudo domiciliar populacional. Rev Saude Publica 2015; 49:51.

11. Brasil. Ministério da Saúde (MS). Projeto SB Brasil 2010: condições de saúde bucal da população brasileira 2010: resultados principais. Brasília: MS; 2011.

12. Peres MA, Ester BPM, Boing AF, Yokota RTC, Malta DC, Peres KG. Desigualdades no acesso e na utilização de serviços odontológicos no Brasil: análise do Sistema de Vigilância de Fatores de Risco e Proteção para Doenças Crônicas por Inquérito Telefônico (VIGITEL 2009). Cad Saude Publica 2012; 28(Supl.):S90-S100.

13. Carvalho JAM, Rodriguez-Wong LL. A transição da estrutura etária brasileira na primeira metade do século XXI. Cad Saude Publica 2008; 24(3):597-605.

14. Martins AMEBL, Barreto SM, Pordeus IA. Características associadas ao uso de serviços odontológicos entre idosos dentados e edentados no Sudeste do Brasil: Projeto SB Brasil. Cad Saude Publica 2008; 24(1):81-92.

15. Martins AMEBL, Barreto SM, Silveira MF, Santa-Rosa TTA, Pereira RD. Autopercepção da saúde bucal entre idosos brasileiros. Rev Saude Publica 2010; 44(5):912922.

16. Roncalli AG, Silva NN, Nascimento AC, Freitas CHSM, Casotti E, Peres KG, Moura L, Peres MA, Freire MCM, Cortes MIS, Vettore MV, Junior MP, Figueiredo N, Goes PSA, Pinto RS, Marques RAM, Moyses SJ, Reis SCGB, Narvai PC. Aspectos metodológicos do Projeto SBBrasil 2010 de interesse para inquéritos nacionais de saúde. Cad Saude Publica 2012; 28(Supl.):s40-s57.
17. Souza JG, Costa Oliveira BE, Martins AM. Contextual and individual determinants of oral health-related quality of life in older Brazilians. Qual Life Res 2017; 26(5):1295-1302.

18. Adulyanon S, Vourapukjaru J, Sheiham A. Oral impacts affecting daily performance in a low dental disease Thai population. Community Dent Oral Epidemiol 1996; 24(6):385-389.

19. Skaret E, Berg E, Raadal M, Kvale G. Factors related to satisfaction with dental care among 23-year olds in Norway. Community Dent Oral Epidemiol 2005; 33(2):150-157.

20. Bedi R, Gulati N, McGrath C. A study of satisfaction with dental services among adults in the United Kingdom. Br Dent J 2005; 198(7):433-437.

21. Moimaz SAS, Marques JAM, Saliba O, Garbin CAS, Zina LG, Saliba NA. Satisfação e percepção do usuário do SUS sobre o serviço público de saúde. Physis 2010; 20(4):1419-1440.

22. Fontes AP, Neri AL. Resiliência e velhice: revisão de literatura. Cien Saude Colet 2015; 20(5):1475-1495.

23. Diez R. A glossary for multilevel analysis. J Epidemiol Community Health 2002; 56(8):588-594.

24. Pinheiro RS, de Torres TZG. Access to oral health services between Brazilian States. Cien Saude Colet 2006,11(4):999-910.

25. Santiago RF, Mendes ACG, Miranda GMD, Duarte PO, Furtado BMASM, Souza WV. Qualidade do atendimento nas Unidades de Saúde da Família no município de Recife: a percepção dos usuários. Cien Saude Colet 2013; 18(1):35-44.

26. Frias AC, Antunes JLF, Junqueira SR, Narvai PC. Determinantes individuais e contextuais da prevalência de cárie dentária não tratada no Brasil. Rev Panam Salud Publica 2007; 22(4):279-285.

27. Peres MA, Peres KG, Frias AC, Antunes JL. Contextual and individual assessmen of dental pain period prevalence in adolescents: a multilevel approach. BMC Oral Health 2010; 10:20.

28. Pinto RS, Abreu MH, Vargas AM. Comparing adult users of public and private dental services in the state of Minas Gerais, Brazil. BMC Oral Health 2014; 14:100.

29. Ferreira CO, Antunes JLF, Andrade FB. Fatores associados à utilização dos serviços odontológicos por idosos brasileiros. Rev Saude Publica 2013; 47(Supl. 3):90-97.

30. Cerutti-Kopplin D, Emami E, Hilgert JB, Hugo FN, Rivaldo E, Padilha DM. Predictors of Satisfaction with Dentures in a Cohort of Individuals Wearing Old Dentures: Functional Quality or Patient-Reported Measures? J Prosthodont 2017; 26(3):196-200.

31. Costa APS, Machado FCA, Pereira ALBP, Carreiro AFP, Ferreira MAF. Technical quality and satisfaction related to full conventional dentures. Cien Saude Colet 2013; 18(2):453-460.

32. Bulgarelli AF, Manço AR. A population of elderly and their satisfaction with their oral health. Cien Saude Colet 2008 ; 13(4):1165-1174.

Artigo apresentado em 01/02/2017

Aprovado em 30/05/2017

Versão final apresentada em 01/06/2017 